\title{
List Colouring Squares of Planar Graphs
}

\author{
Frédéric Havet ${ }^{1}$, Jan van den Heuvel ${ }^{2}$, Colin McDiarmid ${ }^{3}$, and Bruce Reed ${ }^{4}$ \\ ${ }^{1}$ I3S (CNRS/UNSA)-INRIA, Sophia-Antipolis, France \\ ${ }^{2}$ London School of Economics, London, U.K. \\ 3 University of Oxford, Oxford, U.K. \\ 4 McGill University, Montreal, Canada \\ and I3S (CNRS/UNSA)-INRIA, Sophia-Antipolis, France
}

\begin{abstract}
In 1977, Wegner conjectured that the chromatic number of the square of every planar graph $G$ with maximum degree $\Delta \geq 8$ is at most $\left\lceil\frac{3}{2} \Delta\right\rceil+1$. We show that it is at most $\frac{3}{2} \Delta(1+o(1))$.
\end{abstract}

\section{Introduction}

Most of the terminology and notation we use in this note is standard. All our graphs and multigraphs will be finite. A multigraph can have multiple edges; a graph is supposed to be simple. The degree of a vertex is the number of edges incident with that vertex.

We require for all colourings, whether we are discussing vertex, edge or list colouring, to be proper: neighbouring objects must receive different colours. We also always assume that colours are integers, which allows us to talk about the "distance" $\left|c_{1}-c_{2}\right|$ of two colours $c_{1}, c_{2}$.

The chromatic number of a multigraph $G$, denoted $\chi(G)$, is the minimum number of colours required so that we can properly colour its vertices using those colours. If we colour the edge of $G$, we get the chromatic index, denoted $\chi^{\prime}(G)$. The list chromatic number or choice number ch $(G)$ is the minimum value $k$, so that if we give each vertex $v$ of $G$ a list $L(v)$ of at least $k$ colours, then we can find a proper colouring in which each vertex gets assigned a colour from its own private list. The list chromatic index is defined analogously for edges.

\subsection{Colouring the Square of Graphs}

Given a graph $G$, the square of $G$, denoted $G^{2}$, is the graph with the same vertex set as $G$ and with an edge between any two different vertices that have distance at most two in $G$. If $G$ has maximum degree $\Delta$, then a vertex colouring of its square will need at least $\Delta+1$ colours, but the greedy algorithm shows it is always possible with $\Delta^{2}+1$ colours. Diameter two cages such as

email: fhavet@sophia.inria.fr, jan@maths.lse.ac.uk, cmcd@stats.ox.ac.uk, breed@cs.mcgill.ca 
the 5-cycle, the Petersen graph and the Hoffman-Singleton graph show that there exist graphs that in fact require $\Delta^{2}+1$ colours.

Regarding the chromatic number of the square of a planar graph, Wegner [14] posed the following conjecture ( see also the book of Jensen and Toft [6, Section 2.18]), suggesting that for planar graphs far less than $\Delta^{2}+1$ colours suffice.

\section{Conjecture 1.1 ( Wegner [14])}

For a planar graph $G$ of maximum degree $\Delta: \chi\left(G^{2}\right) \leq \begin{cases}7, & \text { if } \Delta=3, \\ \Delta+5, & \text { if } 4 \leq \Delta \leq 7, \\ \left\lceil\frac{3}{2} \Delta\right\rceil+1, & \text { if } \Delta \geq 8 .\end{cases}$

Wegner also gave examples showing that these bounds would be tight. For even $\Delta \geq 8$, these examples are sketched below. This graph has maximum degree $2 k$ and yet all the vertices

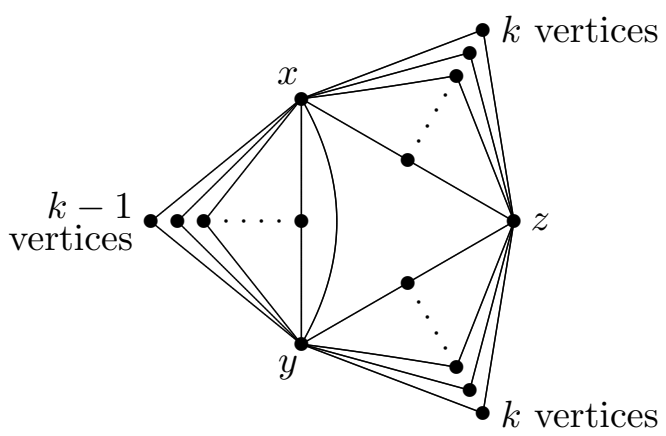

except $z$ are adjacent in its square. Hence to colour these $3 k+1$ vertices, we need at least $3 k+1=\frac{3}{2} \Delta+1$ colours.

Kostochka and Woodall [10] conjectured that for every square of a graph the list chromatic number equals the choose number. This conjecture and Wegner's one imply directly the following conjecture for planar graphs $G: \operatorname{ch}\left(G^{2}\right) \leq \begin{cases}7, & \text { if } \Delta=3, \\ \Delta+5, & \text { if } 4 \leq \Delta \leq 7, \\ \left\lceil\frac{3}{2} \Delta\right\rceil+1, & \text { if } \Delta \geq 8 .\end{cases}$

Wegner also showed that if $G$ is a planar graph with $\Delta=3$, then $G^{2}$ can be 8-coloured. Very recently, Thomassen [13] solved Wegner's conjecture for $\Delta=3$ and Cranston and Kim [3] showed that the square of every connected graph (non necessarily planar) which is subcubic (i.e., with $\Delta \leq 3$ ) is 8-choosable, except for the Petersen graph. However, the 7-choosability of the square of subcubic planar graphs is still open.

The first upper bound on $\chi\left(G^{2}\right)$ in terms of $\Delta$ was obtained by Jonas [7] who showed $\chi\left(G^{2}\right) \leq 8 \Delta-22$. This bound was later improved by Wong [15] to $\chi\left(G^{2}\right) \leq 3 \Delta+5$ and then by Van den Heuvel and McGuinness [5] to $\chi\left(G^{2}\right) \leq 2 \Delta+25$. Better bounds were then obtained for large values of $\Delta$. It was shown that $\chi\left(G^{2}\right) \leq\left\lfloor\frac{9}{5} \Delta\right\rfloor+2$ for $\Delta \geq 749$ by Agnarsson and Halldórsson [1], and that $\chi\left(G^{2}\right) \leq\left\lceil\frac{9}{5} \Delta\right\rceil+1$ for $\Delta \geq 47$ by Borodin et al. [2]. Finally, the best known upper bound so far has been obtained by Molloy and Salavatipour [12]: $\chi\left(G^{2}\right) \leq$ $\left\lceil\frac{5}{3} \Delta\right\rceil+78$. As mentioned in [12], the constant 78 can be reduced for sufficiently large $\Delta$. For example, it was improved to 24 when $\Delta \geq 241$.

In this extended abstract we announce the following theorem. 


\section{Theorem 1.2}

The square of every planar graph $G$ of maximum degree $\Delta$ has list chromatic number at most $(1+o(1)) \frac{3}{2} \Delta$. Moreover, given lists of this size, there is an proper colouring in which the colours on every pair of adjacent vertices of $G$ differ by $\Delta^{1 / 4}$.

The first order term $\frac{3}{2} \Delta$ in Theorem 1.2 is essentially best possible, as the examples above show. On the other hand, the term $\Delta^{1 / 4}$ is probably far from best possible; it was chosen to keep the proof simple. The main point, to our minds, is that this parameter is $o(\Delta)$.

\section{Sketch of the proof of Theorem 1.2}

To prove Theorem 1.2, we need to show that for every $\epsilon>0$, there is a $\Delta_{\epsilon}$ such that for every $\Delta \geq \Delta_{\epsilon}$ we have: for every planar graph $G$ of maximum degree at most $\Delta$, given lists of size $c_{\epsilon}=\left\lfloor\left(\frac{3}{2}+\epsilon\right) \Delta\right\rfloor$ for each vertex $v$ of $G$, we can find the desired colouring.

We proceed by induction on the number of vertices of $G$. Our proof is a recursive algorithm. In each iteration, we split off a set $R$ of vertices of the graph which are easy to handle, recursively colour $G^{2}-R$ (which we can do by the induction hypothesis), and then extend this colouring to the vertices of $R$. In extending the colouring, we must ensure that no vertex $v$ of $R$ receives a colour used on a vertex of $V-R$ which is adjacent to $v$ in $G^{2}$. Thus, we modify the list $L(v)$ of colours available for $v$ by deleting those which appear on such neighbours.

We note that $(G-R)^{2}$ need not be equal to $G^{2}-R$, as there may be vertices of $G$ with a common neighbour in $R$ but no common neighbour in $G-R$. When choosing $R$ we need to ensure that we can construct a planar graph $G_{1}$ on $V-R$ such that $G^{2}-R \subseteq G_{1}^{2}$. We also need to ensure that the connections between $R$ and $V-R$ are limited, so that the modified lists used when list colouring $G^{2}[R]$ are still reasonably large. Finally, we will want $G^{2}[R]$ to have a simple structure so that we can prove that we can list colour it as desired.

We begin with a simple example of such a set $R$. We say a vertex $v$ of $G$ is removable if it has at most $\Delta^{1 / 4}$ neighbours in $G$ and at most two neighbours in $G$ which have degree exceeding $\Delta^{1 / 4}$. We note that if $v$ is a removable vertex with exactly two neighbours $x$ and $y$, then setting $G_{1}=G-v+e$, where $e$ is an edge between $x$ and $y$, we have that $G_{1}$ is a planar graph with $G^{2}-v \subseteq G_{1}^{2}$. On the other hand, if $v$ is a removable vertex with three neighbours, then it must have a neighbour $w$ of degree less than $\Delta^{1 / 4}$. In this case, the graph $G_{2}$ obtained from $G-v$ by adding an edge from $w$ to every other neighbour of $v$ in $G$ is a graph of maximum degree $\Delta$ such that $G^{2}-v \subseteq G_{2}^{2}$. Furthermore, $G_{2}$ is planar as it is obtained from $G$ by contracting the edge $w v$.

Thus, for any removable vertex $v$, we can recursively list colour $G^{2}-v$ using our algorithm. If, in addition, $v$ has at most $c_{\epsilon}-1-2 \Delta^{1 / 2}$ neighbours in $G^{2}$, then our bound on $d_{G^{2}}(v)$ ensures that there will be a colour in $L(v)$ which appears on no vertex adjacent to $v$ in $G^{2}$ and is not within $\Delta^{1 / 4}$ of any colour assigned to a neighbour of $v$ in $G$. To complete the colouring we give $v$ any such colour.

The above remarks show that no minimal counterexample to our theorem can contain a removable vertex of low degree. We are about to describe another, more complicated, reduction we will use. It relies on the following easy result. 


\section{Lemma 2.1}

If $R$ is a set of removable vertices of $G$, then there is a planar graph $G_{1}$ with vertex set $V-R$ and maximum degree $\Delta$ such that $G^{2}-R \subseteq G_{1}^{2}$.

Proof First delete all edges between vertices in $R$. For $v \in R$ of degree two, add an edge between its neighbours. For each $v \in R$ of degree at least three, choose a neighbour of $v$ of degree less than $\Delta^{1 / 4}$ onto which we will contract $v$. Add an edge between the two neighbours of any vertex of degree two in $R$. The degree of a vertex $x$ in the resultant graph $G_{1}$ is at most the maximum of $\Delta^{1 / 2}$ or $d_{G}(x)$.

For any multigraph $H$, we let $H^{*}$ be the graph obtained from $H$ by subdividing each edge exactly once. For each edge $e$ of $H$, we let $e^{*}$ be the vertex of $H^{*}$ which we placed in the middle of $e$ and we let $E^{*}$ be the set of all such vertices. We call this set of vertices corresponding to the vertices of $E^{*}$ the core of $H^{*}$. A removable copy of $H$ is a subgraph of $G$ isomorphic to $H^{*}$ such that the vertices of $G$ corresponding to the vertices of the core of $H^{*}$ are removable.

Note that the subgraph $J$ of $G^{2}$ induced by the core of some copy of $H^{*}$ in $G$ contains a subgraph isomorphic to $L(H)$, the line graph of $H$. So the list chromatic number of $J$ is at least the list chromatic number of $L(H)$. If the copy is removable, then removing the edges of this copy of $L(H)$ from $J$ yields a graph in which the vertices in the core have degree at most $\Delta^{1 / 2}$. Thus, the key to list colouring $J$ will be to list colour $L(H)$. Fortunately, list colouring line graphs is much easier than list colouring arbitrary graphs ( see e.g. $[8,9,11]$ ). In particular, using a sophisticated argument due to Kahn [8], we can prove the following lemma which specifies certain sets of removable vertices which we can use to perform reductions.

\section{Lemma 2.2}

Suppose $R$ is the core of a removable copy of $H^{*}$ in $G$, for some multigraph $H$, such that for any set $X$ of vertices of $H$ and corresponding set $X^{*}$ of vertices of the copy of $H^{*}$, we have that the sum of the degrees in $G-R$ of the vertices in $X^{*}$ exceeds the number of edges of $H$ out of $X$ by at most $\frac{\epsilon|X| \Delta}{10}$. Then, any $c_{\epsilon}$-colouring of $G^{2}-R$ can be extended to a $c_{\epsilon}$-colouring of $G^{2}$.

The following lemma shows that we will indeed be able to find a removable set of vertices which we can use to perform a reduction.

\section{Lemma 2.3}

There is some $\Delta_{0}$ such that any planar graph $G$ of maximum degree $\Delta \geq \Delta_{0}$ contains one of the following:

(A) a removable vertex $v$ which has degree less than $\frac{3}{2} \Delta+\Delta^{1 / 2}$ in $G^{2}$, or

(B) a removable copy of $H^{*}$ with core $R$, for some multigraph $H$ which contains an edge and is such that for any set $X$ of vertices of $H$ we have: the sum of the degrees in $G-R$ of the vertices in $X$ exceeds the number of edges of $H$ out of $X$ by at most $|X| \Delta^{9 / 10}$.

Combining Lemmas 2.1, 2.2, and 2.3 yields Theorem 1.2. Thus, we need only prove the last two of these lemmas. The proof of Lemma 2.3 uses not much more than the fact that planar graphs have low edge-density. The proof of Lemma 2.2 is much more complicated. We follow 
the approach developed by Kahn [8] for his proof that the list chromatic index of a multigraph is asymptotically equal to its fractional chromatic number. We need to modify the proof so it can handle our situation in which we have a graph which is slightly more than a line graph and in which we have lists with fewer colours than he permitted.

Full details will be given in [4].

\section{References}

[1] G. Agnarsson and M.M. Halldórsson, Coloring powers of planar graphs. SIAM J. Discrete Math. 16 (2003), 651-662.

[2] O.V. Borodin, H.J. Broersma, A. Glebov, and J. van den Heuvel, Minimal degrees and chromatic numbers of squares of planar graphs (in Russian). Diskretn. Anal. Issled. Oper. Ser. 1 8, no. 4 (2001), 9-33.

[3] D.W. Cranston and S.-J. Kim, List-coloring the square of a subcubic graph. Manuscript, 2006 .

[4] F. Havet, J. van den Heuvel, C. McDiarmid, and B. Reed, List colouring squares of planar graphs. In preparation.

[5] J. van den Heuvel and S. McGuinness, Coloring the square of a planar graph. J. Graph Theory 42 (2003), 110-124.

[6] T.R. Jensen, B. Toft, Graph Coloring Problems. John-Wiley \& Sons, New York, 1995.

[7] T.K. Jonas, Graph coloring analogues with a condition at distance two: $L(2,1)$-labelings and list $\lambda$-labelings. Ph.D. Thesis, University of South Carolina, 1993.

[8] J. Kahn, Asymptotics of the list-chromatic index for multigraphs. Random Structures Algorithms 17 (2000), 117-156.

[9] P.M. Kayll, Asymptotically good choice numbers of multigraphs. Ars Combin. 60 (2001), 209-217.

[10] A.V. Kostochka and D.R. Woodall, Choosability conjectures and multicircuits. Discrete Math. 240 (2001), 123-143.

[11] M. Molloy and B. Reed, Graph colouring and the probabilistic method. Algorithms and Combinatorics 23. Springer-Verlag, Berlin, 2002.

[12] M. Molloy and M.R. Salavatipour, A bound on the chromatic number of the square of a planar graph. J. Combin. Theory Ser. B 94 (2005), 189-213.

[13] C. Thomassen, The square of a planar cubic graph is 7-colorable. Manuscript, 2006.

[14] G. Wegner, Graphs with given diameter and a coloring problem. Technical Report, University of Dortmund, 1977.

[15] S.A. Wong, Colouring graphs with respect to distance. M.Sc. Thesis, Department of Combinatorics and Optimization, University of Waterloo, 1996. 